\title{
Imaging Cosmic Ray Particles with a Light Microscope Scientific Digital Camera
}

\author{
George McNamara
}

University of Texas M.D. Anderson Cancer Center, 1515 Holcombe Boulevard, Houston, TX 77030

geomcnamara@earthlink.net

\section{Introduction}

Cosmic ray particles were discovered by Victor Hess on August 7, 1912, leading to his co-winning the Nobel Prize in Physics in 1936 [1, 2]. Initially thought to be rays (photons), in the 1920s they were recognized as being charged particles [1]. I describe in this article how I acquire and process images of cosmic ray particles (CRPs) on a microscope with a digital camera in the imaging core facility I manage.

Of relevance to light microscopists, a research-grade light microscope is typically equipped with a charge-coupled device (CCD) camera that is struck by a cosmic ray particle about once every 30 seconds. This is not a big deal for an $\mathrm{N}=1$ experiment of one green/red fluorescent micrograph with exposure times of less than 1 second per channel. It could lead to confusion, however, when acquiring a long time series involving low-lightlevel fluorescence imaging such as single-molecule fluorescence tracking or in precision localization microscope (PLM) techniques, such as PALM, STORM, FPALM, and related techniques [3-7].

\section{Methods and Materials}

The figures accompanying this manuscript were all processed from a 5,380-minute $(322,800$ seconds) data set acquired over the 2012 Labor Day weekend (Friday August 31 to Tuesday September 4, 2012). I used a Hamamatsu ORCA-II ERG digital CCD camera. The sensor was cooled to $-60^{\circ} \mathrm{C}$, dark current was 0.0045 electrons/pixel/second, and the exposure time was 2 hours. For this dataset, I acquired 538 ten-minute exposures in one time series. The ORCA-II ERG camera uses the same ER-150 interline $\mathrm{CCD}$ as in the popular ORCA-ER, with the latter cooled to $-20^{\circ} \mathrm{C}$, dark current 0.1 electrons/pixel/second, and maximum exposure time 10 seconds. I have acquired weekend CRP datasets with my core's ORCA-ERs, but then typically used $2 \times 2$ binning to make the file size more reasonable when acquiring $\sim 32,000$ ten-second ( $\sim 320,000$ seconds) exposures over a holiday weekend. Thus, one advantage of the ORCA-II ERG's exceptional cooling is simply that more events can be captured in fewer images and at full sensor resolution $(1344 \times 1024$ pixels, sensor area $8.67 \times 6.60 \mathrm{~mm})$. The Labor Day dataset was acquired in ORCA-II ERG 12-bit mode, though in retrospect 14-bit mode could have provided additional information. (Either way the data were saved to a 16-bit Zeiss ZVI file and later exported as a 16-bit TIFF.) Image acquisition was performed in time-series mode using Zeiss Axiovision 4.8.2.

The ORCA-II ERG camera is mounted on a Zeiss Axiovert $200 \mathrm{M}$ inverted motorized microscope with objective lens turret, filter cube turret, objective lens nosepieces, and camera/eyepieces ports motorized. The Zeiss FluoArc mercury arc lamp and halogen transmission lamps were off. The objective lens turret was on a blocked (lens cap) position. The ORCA-II ERG camera was on the left camera port, and I selected the microscope light path to go to the right camera port (blocked with a light tight cap). I selected the Zeiss Cy5 filter set, covered the stage with a black clipboard, shielded the microscope from the computer monitor with two additional clipboards, and placed a box over the eyepiece port.
After starting the time series, I turned off the HP ZR30w monitor, turned off all lighting (a tungsten reading light on a side table and the overhead fluorescent lighting), closed the lab door, and placed a "do not disturb" sign outside. The acquisition PC was a Dell Optiplex 960 with Windows XP (32-bit operating system).

At the end of the time series, the dataset was saved to Zeiss ZVI file, it was exported to 16-bit TIFF format using Axiovision (the free Axiovision LE also enabled exporting), and then the TIFF series was opened with MetaMorph (64-bit) version 7.7.3 [8] on a Dell Optiplex 960 with Windows 7 (64-bit O.S., 16 Gb RAM). I then resaved the data in MetaMorph Stack format. The ten-minute exposures have an offset + dark current ranging from 560-630 corner to corner, so I used the Stack Arithmetic: Median command to produce an estimate of this background. I then subtracted this median image from every plane in the stack and saved the result as my "background subtracted" stack. Using the median image is not a perfect method to eliminate background, so I (somewhat arbitrarily) selected 40 intensity levels as my lower threshold in deciding what "events" to count. By performing Stack Arithmetic: Maximum on the background subtracted stack, I made a single image with all the "events." This is the same operation that I use for "maximum projections" of a fluorescence Z-series or time series. Given the sparseness of the events, this was a reasonable approach, but users who would prefer to count plane-by-plane can download the dataset from my web site [9].

\section{Results}

MetaMorph's Integrated Morphometry Analysis ("IMA") command, with a threshold minimum of 50, counted 1,486 "objects" when the IMA object was set to be at least 2 pixels in size. A threshold minimum of 40 counted 1,742; a threshold minimum of 30 counted 2,051 objects; a threshold minimum of 25 counted 2,273 objects; and a threshold minimum of 20 yielded many single pixels from the residual background noise. This is as expected from working with $1344 \times 1024 \times 538=740,425,728$ pixels of data and having chosen to use a median of each pixel from 538 planes for subtraction. For a 5,380-minute dataset, I might have expected $\sim 10,000$ "events." The apparent undercount might be due to many events producing only weak "hits" above the camera floor. Figures 1 and 2 show the results. The cosmic ray particle tracks are revealed in these figures.

Because of space limitations, I will not go into the intensity distribution of the events. I am posting the data online [9] to enable readers to evaluate this dataset and consider acquiring and evaluating your own data.

\section{Discussion}

Considering the care and time involved, this type of experiment may not have been accomplished previously on a light microscope with a digital camera. The image acquired here was not intended to make a publishable image of a cosmic ray, but rather to act as a cautionary for people who are doing low-light-level and single-molecule imaging. 


\section{Breakthroughs in Nanotechnology}

Image, characterize, manipulate, fabricate...at the nanoscale!

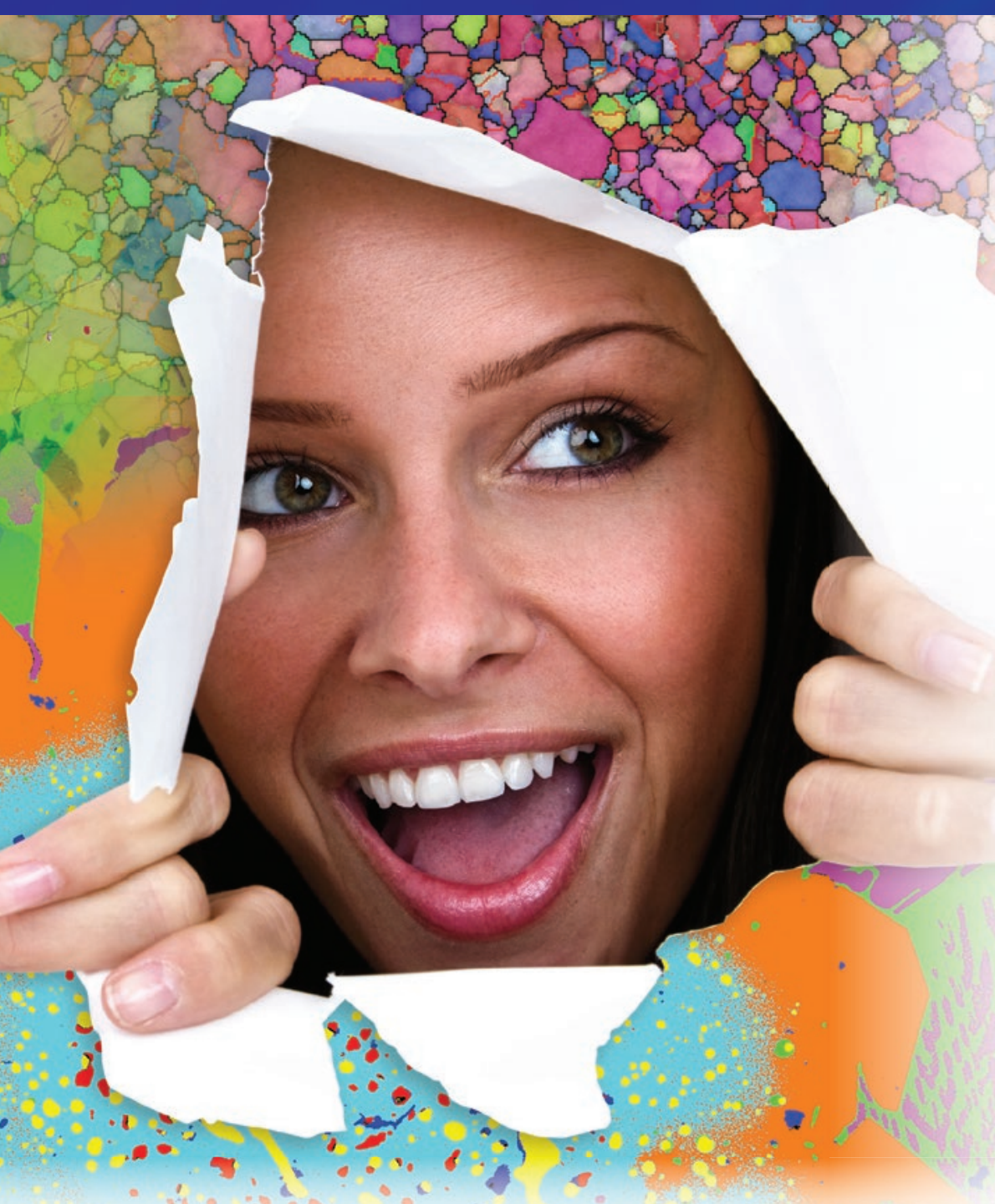

Breakthroughs - it's what we do.

In the last year we've launched

many new products:

$\mathbf{X}-\mathbf{M a x}^{\mathbf{N}} 20,50,80$

and $150 \mathrm{~mm}^{2}$ SDD

AutoPhaseMap phase analysis

LayerProbe thin film

and coating analysis

NordlysMax² high speed

EBSD detector

OmniProbe $4001 \mathrm{~nm}$ resolution

nanomanipulator

OmniGIS II single-needle

multiple-gas injection system

Asylum Research MFP-3D

Origin AFM

Imagine what we'll be showing at M\&M 2013 in Indianapolis!

Visit our booth,

See you there.

see something amazing.

Book your demos now! www.oxford-instruments.com/MandM

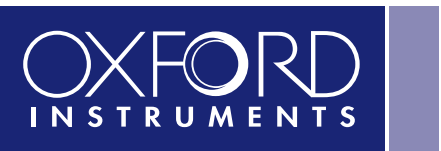

The Business of Science ${ }^{\circledast}$ 


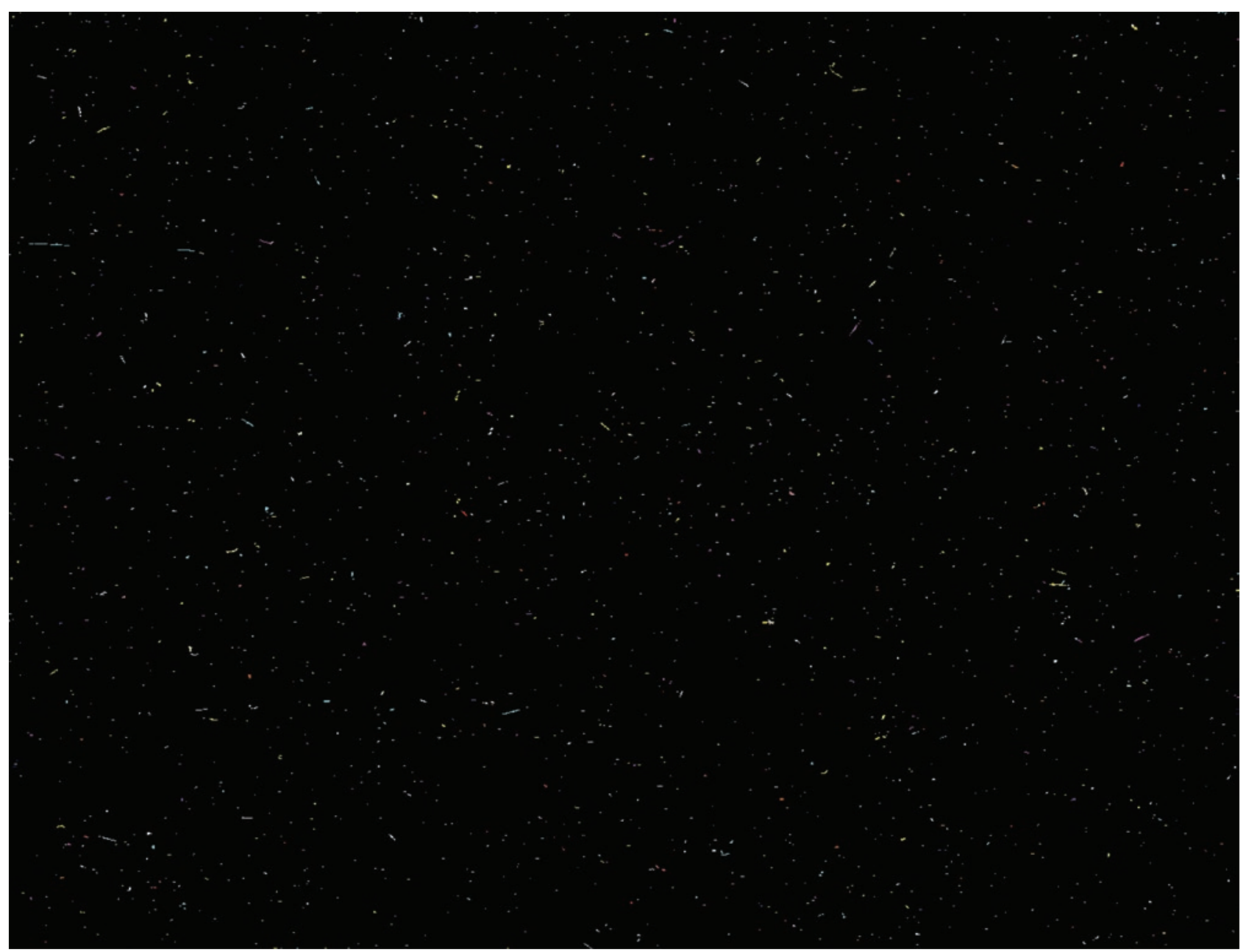

Figure 1: Cosmic ray particle tracks revealed in a five-day-long exposure. Color image combines red 001-180, green 181-360, and blue 361-538 planes into a contrasted 24-bit.tif image.

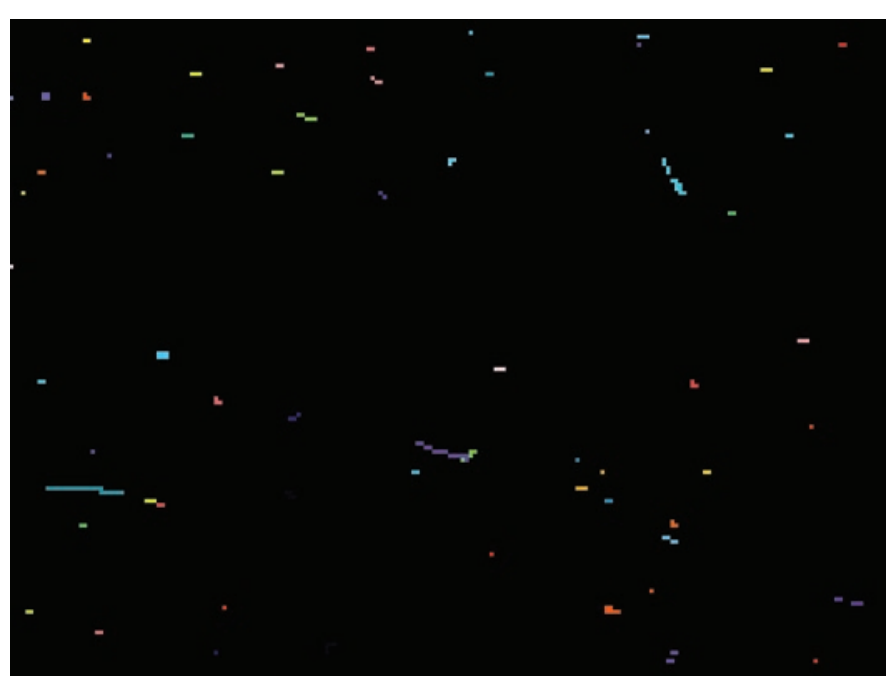

Figure 2: Detail from near the center of Figure 1 showing cosmic ray particle tracks.

\section{Conclusion}

Long-term images may contain details from cosmic rays that could be taken for image information. These images show artifacts of dots and tracks that could be for real data in super-resolution or single-molecule imaging.

\section{References}

[1] http://en.wikipedia.org/wiki/Cosmic_ray

[2] http://www.nobelprize.org/nobel_prizes/physics/laureates /1936/

[3] E Betzig, GH Patterson, R Sougrat, OW Lindwasser, S Olenych, JS Bonifacino, MW Davidson, J LippincottSchwartz, and HF Hess, Science 313 (2006) 1642-45.

[4] MJ Rust, M Bates, and X Zhuang, Nat Methods 3 (2006) 793-95.

[5] ST Hess, TP Girirajan, and MD Mason, Biophys J 91 (2006) $4258-72$.

[6] MF Juette, TJ Gould, MD Lessard, MJ Mlodzianoski, BS Nagpure, BT Bennett, ST Hess, and J Bewersdorf, Nat Methods 5 (2008) 527-29.

[7] Vutara SR-200 super-resolution microscope.

[8] The MetaMorph 7.7.3 is the author's personal license from an "Amazing image" offer that Molecular Devices ran several years ago. My thanks to the M.D. marketing department for this license.

[9] Data available in ZIP file at http://works.bepress.com /gmcnamara/24/. 

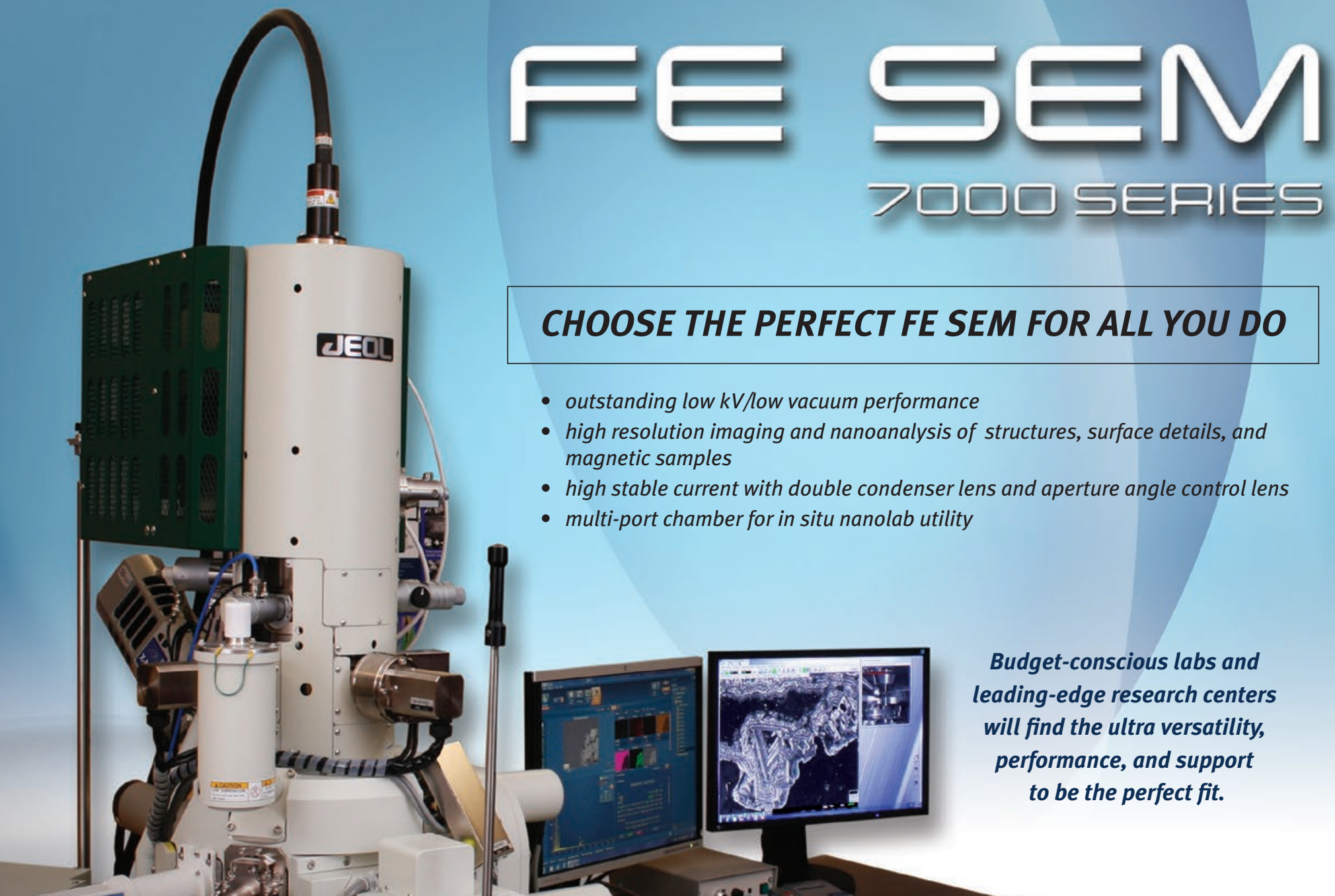

\section{CHOOSE THE PERFECT FE SEM FOR ALL YOU DO}

- outstanding low $\mathrm{kV} / \mathrm{low}$ vacuum performance

- high resolution imaging and nanoanalysis of structures, surface details, and magnetic samples

- high stable current with double condenser lens and aperture angle control lens

- multi-port chamber for in situ nanolab utility
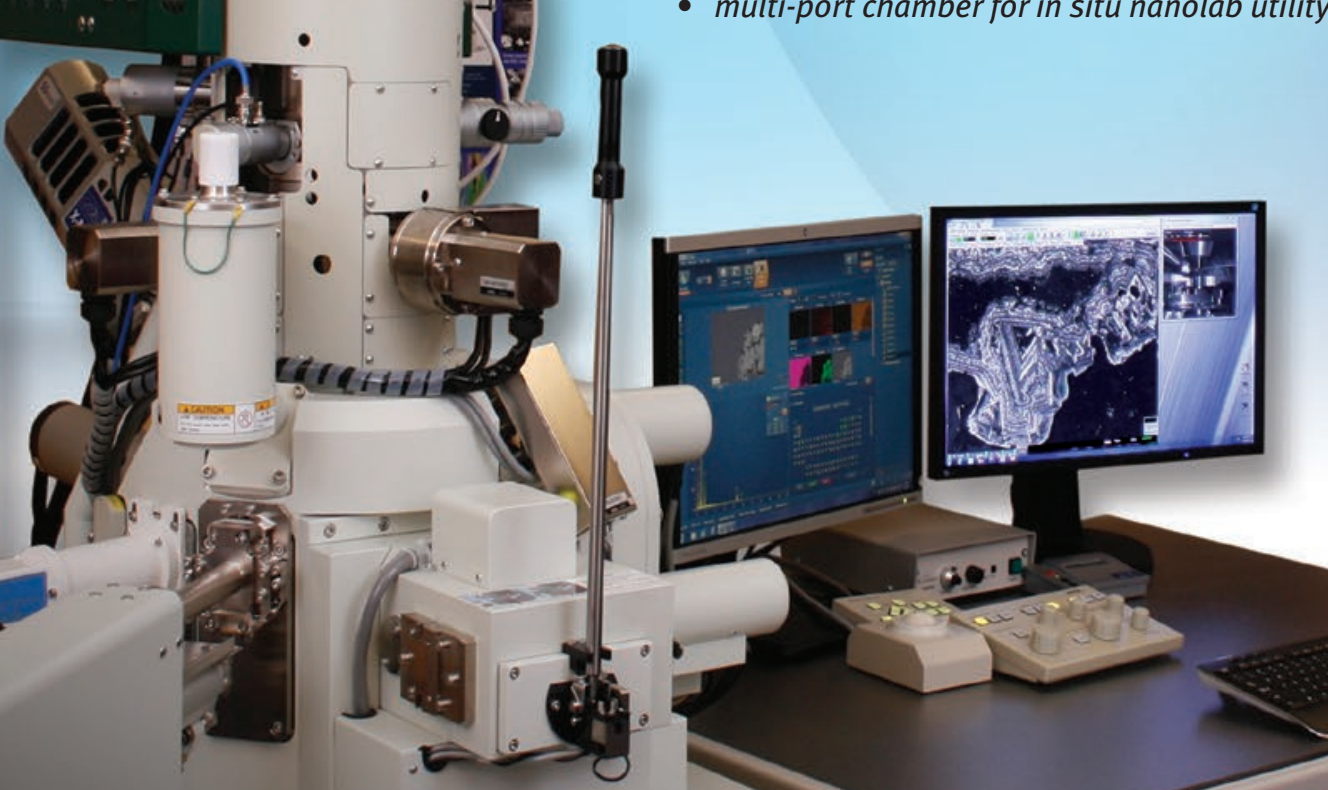

Budget-conscious labs and leading-edge research centers will find the ultra versatility, performance, and support to be the perfect fit.
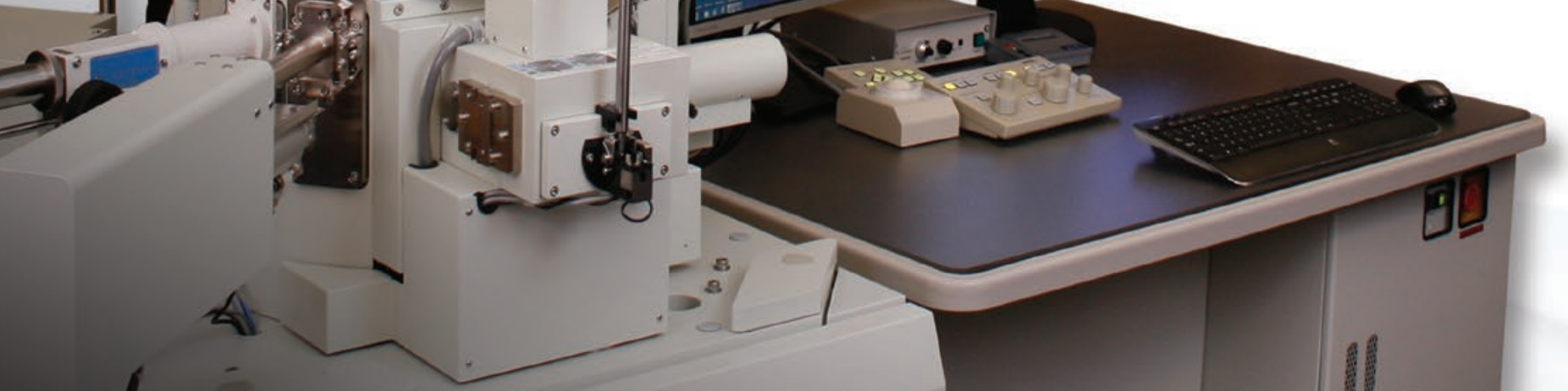